\title{
Intercomparison of Techniques to Correct for Attenuation of C-Band Weather Radar Signals
}

\author{
Eugenio Gorgucci AND GianFranco ScARCHILLI \\ Istituto di Fisica dell'Atmosfera, CNR, Rome, Italy \\ V. ChandRasekAR \\ Colorado State University, Fort Collins, Colorado
}

P. F. MEISCHNER AND M. HAGEN

DLR, Institut für Physik der Atmosphäre, Oberpfaffenhofen, Germany

(Manuscript received 23 May 1997, in final form 30 December 1997)

\section{ABSTRACT}

\begin{abstract}
Quantitative application of radar measurements at $\mathrm{C}$ band requires correction for attenuation. Algorithms to correct for attenuation and differential attenuation are evaluated based on theoretical analysis as well as radar data. The error structure of three different attenuation correction algorithms based on (a) reflectivity, (b) reflectivity and differential reflectivity, and (c) specific differential propagation phase is analyzed. The error structure of two algorithms to correct the differential attenuation based on (a) reflectivity and differential reflectivity, and (b) specific differential propagation phase is presented. Data from the polarimetric C-band Doppler radar POLDIRAD operated by DLR (Germany) are utilized to intercompare the attenuation and differential attenuation correction algorithms. Radar data and theoretical analysis show that the attenuation correction algorithm using reflectivity and differential reflectivity agrees well with the attenuation correction algorithm based on specific differential phase. Similarly, radar data and theoretical analysis indicate that the algorithms to correct for differential attenuation compare well with each other. In addition the fractional standard error of comparison between the algorithms to correct for attenuation and differential attenuation is in good agreement with theoretical results, providing an indirect verification of the accuracies of the algorithms.
\end{abstract}

\section{Introduction}

C-band radar systems are widely used in Europe for meteorological applications. S-band weather radar systems are commonly used in the United States for operational applications requiring long-range coverage such as few hundred kilometers. Radars that operate at higher frequencies offer the advantage of lower cost resulting from smaller antenna size compared to lowerfrequency radars having the same spatial resolution. However, the resulting spatial resolution at lower cost is offset by attenuation problems. Quantitative interpretation of echo powers at C-band frequencies requires correction for attenuation to avoid errors in estimating precipitation. Attenuation correction for C-band radars can be done with different methods depending on the type of measurements involved. Conventional attenua-

Corresponding author address: Dr. Eugenio Gorgucci, Istituto di Fisica dell'Atmosfera, Via del Fosso del Cavaliere, 00133 Rome, Italy.

E-mail: gorgucci@radar.ifa.rm.cnr.it tion correction procedures involve a $Z-\alpha$ relation where the specific attenuation $(\alpha)$ is related to the reflectivity factor $(Z)$ by a power law. However, the absence of a unique relation made them difficult to use (Aydin et al. 1989). Aydin et al. (1989) introduced an attenuation correction procedure for dual-polarization radars parameterizing the ratio of specific attenuation and reflectivity factor $(\alpha / Z)$ in terms of the differential reflectivity $\left(Z_{\mathrm{DR}}\right)$. Bringi et al. (1990) examined an attenuation correction procedure based on the relationship between the specific differential phase $\left(K_{\mathrm{DP}}\right)$ and specific attenuation.

Excessively attenuated radar echoes can be spotted on reflectivity maps such as range streaks. However, when attenuation is not excessive (such as a few decibels), the effects are difficult to observe. C-band radar signals fall under this category, where the typical attenuation levels encountered are neither excessive (several tens of decibels) nor negligible (less than $0.15 \mathrm{~dB}$ ). This poses a problem for applications involving quantitative measurement of reflectivity. Scarchilli et al. (1993) have shown from theoretical studies that when the total attenuation is small $(\sim 1 \mathrm{~dB})$ the attenuation correction process introduces errors, which outweigh the 
benefit of the attenuation correction. Gorgucci et al. (1995) introduced a simplified version of the attenuation correction procedure suggested by Aydin et al. (1989) by directly parameterizing the specific and differential attenuation in terms of radar measurements such as $Z$ and $Z_{\mathrm{DR}}$.

In this paper we conduct a detailed study intercomparing the various attenuation correction procedures using theoretical simulations as well as data collected by the C-band polarization diversity radar POLDIRAD operated by Deutsche Forschungsanstalt für Luft- und Raumfahrt (DLR) (Germany). The data reported in this paper were collected as part of a collaborative program between the Radar Meteorology Group of the Institute of Atmospheric Physics (IFA) of the National Research Council (CNR) of Italy and the DLR Institute of Atmospheric Physics of Germany.

Our paper is organized as follows: Section 2 presents the various attenuation correction procedures. In section 3 we conduct an error study of the attenuation correction procedures considered. Section 4 describes the dataset and instrumentation. In section 5 the intercomparison of the attenuation correction estimates from radar data is presented. Section 6 summarizes the important results of this paper.

\section{Attenuation correction procedures at $\mathbf{C}$ band}

Reflectivity measurements at $\mathrm{C}$ band are affected by attenuation of radar signals passing through precipitation that exists between the radar and the measurement cell. Differential reflectivity measurements at $\mathrm{C}$ band are similarly affected by the differential attenuation between horizontally $(\mathrm{H})$ and vertically $(\mathrm{V})$ polarized waves. The distributions of raindrop size and shape determine the values of the radar observables as, for example, the reflectivity factor $Z$, the differential reflectivity $Z_{\mathrm{DR}}$, and the specific attenuation $\alpha$. The gamma distribution model adequately describes the natural variation of the raindrop size distribution (RSD). This is given by

$$
N(D)=N_{0} D^{\mu} \exp \left[\frac{-(3.67+\mu) D}{D_{0}}\right],
$$

where $N_{0}, D_{0}$, and $\mu$ are the parameters of the RSD and $D_{0}$ is the median volume diameter (Ulbrich 1983). The equilibrium shape of a raindrop falling at its terminal fall speed is determined by the balance between the forces due to surface tension, hydrostatic pressure, and aerodynamic pressure from airflow around the drop. The shape of a raindrop can be approximated by an oblate spheroid with the axis ratio $(b / a)$ of the drop approximated by the relationship

$$
\frac{b}{a}=1.03-0.062 D_{e},
$$

where $D_{e}$ is the equivolumetric spherical diameter of a raindrop in millimeters, and $a$ and $b$ are the major and minor axes of the drop, respectively (Beard and Chuang 1987; Chandrasekar et al. 1988). The radar measurements such as the reflectivity factor at horizontal and vertical polarization $Z_{H, V}$ and $Z_{\mathrm{DR}}$ can be expressed in terms of the RSD as follows:

$$
Z_{H, V}=\frac{\lambda^{4}}{\pi^{5}|K|^{2}} \int \sigma_{H, V}(D) N(D) d D,
$$

where $\sigma_{H, V}$ are the radar cross sections of raindrops corresponding to $\mathrm{H}$ and $\mathrm{V}$ polarizations,

$$
Z_{\mathrm{DR}}=10 \log \left(\frac{Z_{H}}{Z_{V}}\right)
$$

(Seliga and Bringi 1976). We refer to the article by Bringi and Hendry (1990) for details regarding polarization diversity measurements.

The specific attenuation at horizontal polarization, $\alpha_{H}$ (attenuation per unit length), and the specific differential attenuation, $\alpha_{D}$ (differential attenuation per unit length), between the two polarization states $\mathrm{H}$ and $\mathrm{V}$ are related to the RSD as follows (Bringi et al. 1990):

$$
\alpha_{H, V}=4.343 \times 10^{-3} g_{m} \int f_{H, V} N(D) d D
$$

and

$$
\alpha_{D}=\alpha_{H}-\alpha_{V},
$$

where $f_{H, V}$ are the forward scattering amplitudes at $\mathrm{H}$ and $\mathrm{V}$ polarization states, respectively, and $I m$ refers to imaginary part of a complex number. Scarchilli et al. (1993) have studied the variability of $\alpha_{H}$ and $\alpha_{D}$ as a function of rainfall rate at $\mathrm{C}$-band frequencies. Their results show that specific attenuation rates can be as high as $0.5 \mathrm{~dB} \mathrm{~km}^{-1}$ and $\alpha_{D}$ can be as high as $0.15 \mathrm{~dB}$ $\mathrm{km}^{-1}$. These results show that the absolute attenuation through large rain cells could be easily several decibels in magnitude, while comparable values of differential attenuation could also reach as much as a few decibels.

There are essentially three different ways to correct for the attenuation caused by precipitation. The first is based on the conventional procedure, which involves estimation of attenuation using a power-law relation to approximate $\alpha_{H}$ in term of $Z_{H}$ (Hildebrand 1978). The second procedure utilizes polarimetric measurements and was introduced by Aydin et al. (1989) to correct for the attenuation in $\mathrm{C}$ band parameterizing the relationship between the ratio $\left(\alpha_{H} / Z_{H}\right)$ and $Z_{\mathrm{DR}}$. Gorgucci et al. (1995) simplified this procedure to directly estimate $\alpha_{H}$ and $\alpha_{D}$ using $Z_{H}$ and $Z_{\mathrm{DR}}$. Bringi et al. (1990) and Scarchilli et al. (1993) examined a third procedure to correct for attenuation and differential attenuation using $K_{\mathrm{DP}}$ measurements. In this paper we conduct a detailed study of the error structure of all these procedures and also present an intercomparison between the different techniques. 
TABLE 1 . The variation of the coefficients in $\hat{\alpha}_{H}$ with temperature.

\begin{tabular}{rccc}
\hline \hline$T\left({ }^{\circ} \mathrm{C}\right)$ & $C_{H}$ & $a_{1}$ & $b_{1}$ \\
\hline 0.5 & $9.89 \times 10^{-6}$ & 0.95 & -0.130 \\
2.0 & $9.03 \times 10^{-6}$ & 0.96 & -0.124 \\
5.0 & $7.78 \times 10^{-6}$ & 0.97 & -0.119 \\
10.0 & $6.31 \times 10^{-6}$ & 0.97 & -0.104 \\
20.0 & $4.02 \times 10^{-6}$ & 0.98 & -0.080 \\
\hline
\end{tabular}

The specific attenuation $\alpha_{H}$ and differential attenuation $\alpha_{D}$ can be parameterized in terms of $Z_{H}$ and $Z_{\mathrm{DR}}$ as

$$
\begin{aligned}
& \hat{\alpha}_{H}=C_{H} Z_{H}^{\alpha_{1}} 10^{b_{1} Z_{\mathrm{DR}}} \\
& \hat{\alpha}_{D}=C_{D} Z_{H}^{\alpha_{2}} 10^{b_{2} Z_{\mathrm{DR}}} .
\end{aligned}
$$

The coefficients $C_{H}, C_{D}, a_{1}, a_{2}, b_{1}$, and $b_{2}$ vary with temperature, but not extensively. Tables 1 and 2 show the coefficients in the parameterizations (7) and (8) as a function of temperature. The parameterization for $10^{\circ} \mathrm{C}$ is used in this paper (to fit the environment of the radar data presented). Figure 1a shows a scatterplot of $\hat{\alpha}_{H}$ versus $\alpha_{H}$, whereas Fig. $1 \mathrm{~b}$ shows $\hat{\alpha}_{D}$ versus $\alpha_{D}$ for different RSDs (Ulbrich 1983). Figures 1a and 1b show the ability of the parameterization in (7) and (8) to estimate the specific attenuation and differential attenuation. The estimates $\hat{\alpha}_{H}$ and $\hat{\alpha}_{D}$ follow the actual attenuation values fairly well, with a very narrow scatter and the correlation coefficients are 0.998 and 0.991 , respectively. For completion we have also parameterized $\alpha_{H}$ in terms of the reflectivity factor $Z_{H}$ only as

$$
\hat{\alpha}_{Z}=C_{Z} Z_{H}^{\alpha_{3}} \text {. }
$$

Figure 2 shows a scatterplot of $\hat{\alpha}_{Z}$ versus $\alpha_{H}$, and Table 3 shows the variability of the coefficients in (9), which are obtained with the same technique as in (7) and (8), as a function of temperature. We can see from a comparison of Fig. 1a with Fig. 2 that the algorithm given by (7) tracks attenuation significantly better than the algorithm given by (9) using reflectivity only.

Attenuation and differential attenuation cumulatively increase with the range. Therefore, echoes from cells close to the radar are not attenuated as much as the echoes from storm cells farther from the radar. It can be assumed that the closest echo is not attenuated and the attenuation cumulatively adds up from that point. Therefore, the attenuation is estimated from the first range echo point and then the reflectivities are corrected sequentially in range. A cumulative procedure in range is used similar to the one used by Aydin et al. (1989) to correct for attenuation and differential attenuation. The algorithm for attenuation is as follows:

$$
\left(\hat{Z}_{H}\right)_{n}=\left(Z_{H}^{m}\right)_{n}+\sum_{i=1}^{n-1}\left(\hat{\alpha}_{H}\right)_{i} \Delta r,
$$

where $\left(\hat{Z}_{H}\right)_{n}$ is the reflectivity at range bin $n$ corrected for attenuation, $\left(Z_{H}^{m}\right)_{n}$ is the measured reflectivity at range bin $n, \Delta r$ is the range gate spacing, and $\left(\hat{\alpha}_{H}\right)_{i}$ is the estimate (7) of the specific attenuation at range bin
TABLE 2 . The variation of the coefficients in $\hat{\alpha}_{D}$ with temperature.

\begin{tabular}{rccc}
\hline \hline$T\left({ }^{\circ} \mathrm{C}\right)$ & $C_{D}$ & $a_{2}$ & $b_{2}$ \\
\hline 0.5 & $6.47 \times 10^{-7}$ & 1.02 & -0.052 \\
2.0 & $6.84 \times 10^{-7}$ & 1.02 & -0.050 \\
5.0 & $6.62 \times 10^{-7}$ & 1.01 & -0.044 \\
10.0 & $5.86 \times 10^{-7}$ & 1.02 & -0.030 \\
20.0 & $5.03 \times 10^{-7}$ & 1.01 & -0.011 \\
\hline
\end{tabular}
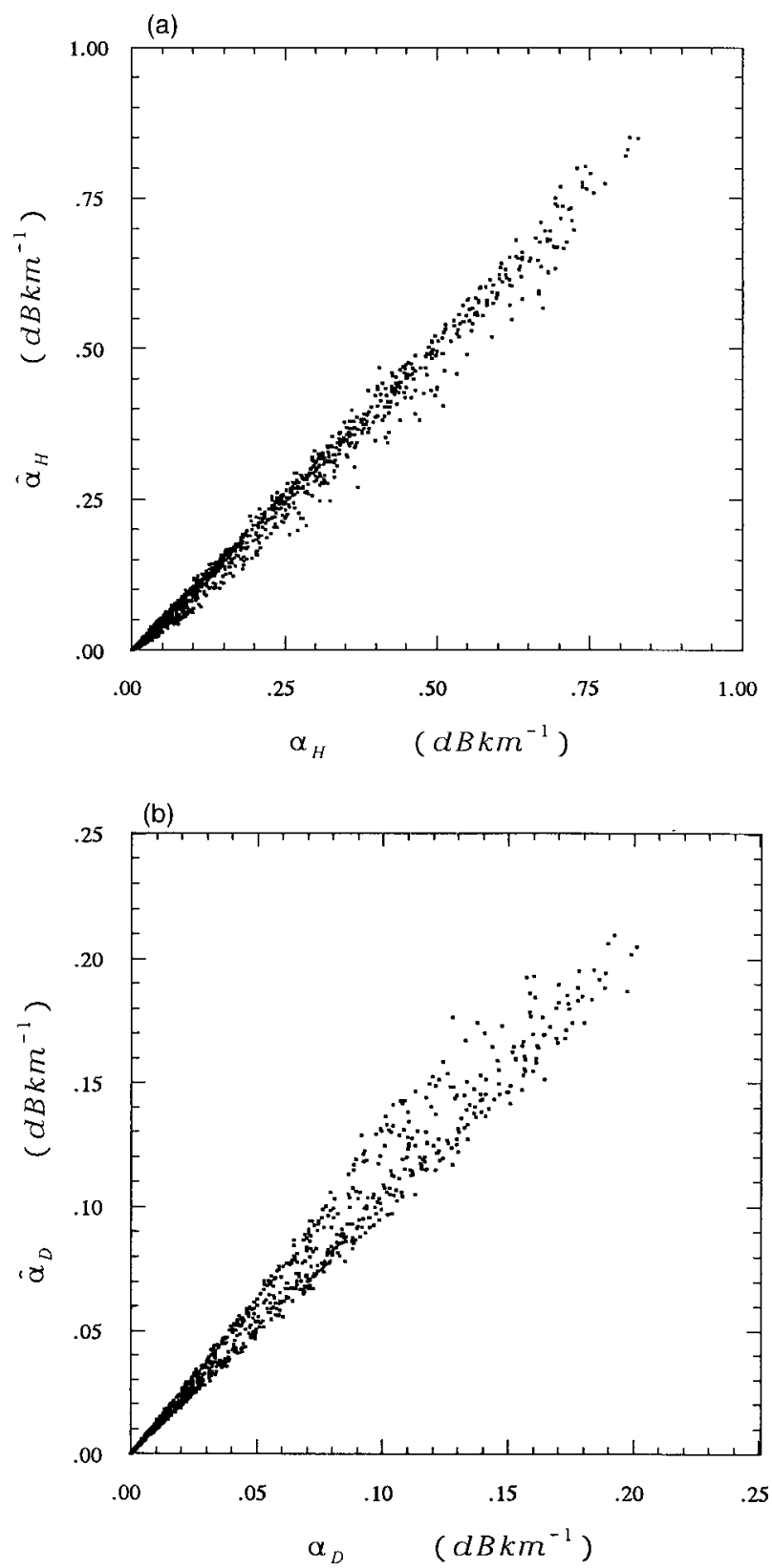

FIG. 1. (a) Scatterplot of the estimate of specific attenuation $\left(\hat{\alpha}_{H}\right)$ using $Z_{H}$ and $Z_{\mathrm{DR}}$ as a function of specific attenuation $\left(\alpha_{H}\right)$ for different RSDs. (b) Scatterplot of the estimate of specific differential attenuation $\left(\hat{\alpha}_{D}\right)$ using $Z_{H}$ and $Z_{\mathrm{DR}}$ as a function of specific differential attenuation $\left(\alpha_{D}\right)$ for different RSDs. 


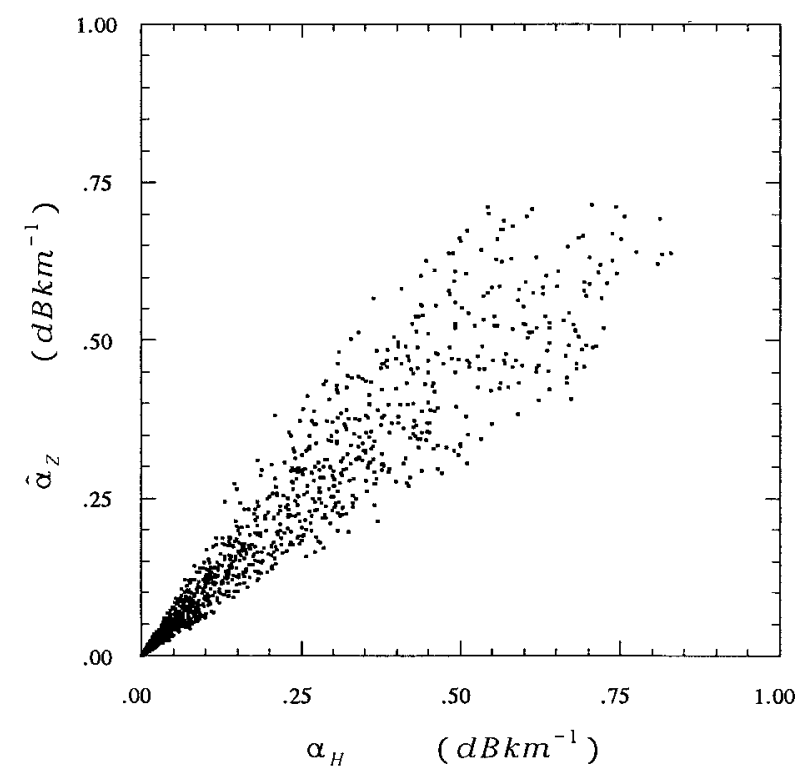

FIG. 2. Scatterplot of the estimate of specific attenuation $\left(\hat{\alpha}_{z}\right)$ using $Z_{H}$ as a function of specific attenuation $\left(\alpha_{H}\right)$ for different RSDs.

$i$. It should be noted here that reflectivity estimate at range $\left(\hat{Z}_{H}\right)_{i}$ is corrected for attenuation up to $(i-1)$ range bins. Similarly the differential reflectivity measurements can be corrected as

$$
\left(\hat{Z}_{\mathrm{DR}}\right)_{n}=\left(Z_{\mathrm{DR}}^{m}\right)_{n}+\sum_{i=1}^{n-1}\left(\hat{\alpha}_{D}\right)_{i} \Delta r
$$

where $\left(\hat{Z}_{\mathrm{DR}}\right)_{n}$ is the estimate of differential reflectivity at range bin $n$ corrected for differential attenuation, $\left(Z_{\mathrm{DR}}^{m}\right)_{n}$ is the measured differential reflectivity at range bin $n$, and $\left(\hat{\alpha}_{D}\right)_{i}$ is the estimate (8) of the specific differential attenuation at range $i$. Similar to the parameterization in terms of $Z_{H}$ and $Z_{\mathrm{DR}}, \alpha_{H}$ and $\alpha_{D}$ can also be parameterized in terms of $K_{\mathrm{DP}}$ as follows:

$$
\hat{\alpha}_{H}^{*}=C_{H}^{*} K_{\mathrm{DP}}
$$

and

$$
\hat{\alpha}_{D}^{*}=C_{D}^{*} K_{\mathrm{DP}}
$$

Table 4 shows the coefficients in the parameterizations (12) and (13) at $\mathrm{C}$ band as a function of temperature. Figure 3 a shows a scatterplot of $\hat{\alpha}_{H}^{*}$ versus the true value of $\alpha_{H}$, whereas Fig. 3b shows $\hat{\alpha}_{D}^{*}$ versus the true value of $\alpha_{D}$ for different RSDs.

The range-cumulative two-way differential phase

TABLE 3. The variation of the coefficients in $\hat{\alpha}_{z}$ with temperature.

\begin{tabular}{rcc}
\hline \hline$T\left({ }^{\circ} \mathrm{C}\right)$ & $C_{z}$ & $a_{3}$ \\
\hline 0.5 & $7.16 \times 10^{-5}$ & 0.73 \\
2.0 & $4.36 \times 10^{-5}$ & 0.77 \\
5.0 & $4.70 \times 10^{-5}$ & 0.76 \\
10.0 & $2.16 \times 10^{-5}$ & 0.82 \\
20.0 & $1.69 \times 10^{-5}$ & 0.83 \\
\hline
\end{tabular}

TABLE 4. The variation of the coefficients in $\hat{\alpha}_{H}^{*}$ and $\hat{\alpha}_{D}^{*}$ with temperature.

\begin{tabular}{rcc}
\hline \hline$T\left({ }^{\circ} \mathrm{C}\right)$ & $C_{H}^{*}$ & $C_{D}^{*}$ \\
\hline 0.5 & $7.17 \times 10^{-2}$ & $1.44 \times 10^{-2}$ \\
2.0 & $6.98 \times 10^{-2}$ & $1.40 \times 10^{-2}$ \\
5.0 & $6.53 \times 10^{-2}$ & $1.30 \times 10^{-2}$ \\
10.0 & $5.88 \times 10^{-2}$ & $1.23 \times 10^{-2}$ \\
20.0 & $4.85 \times 10^{-2}$ & $1.10 \times 10^{-2}$ \\
\hline
\end{tabular}
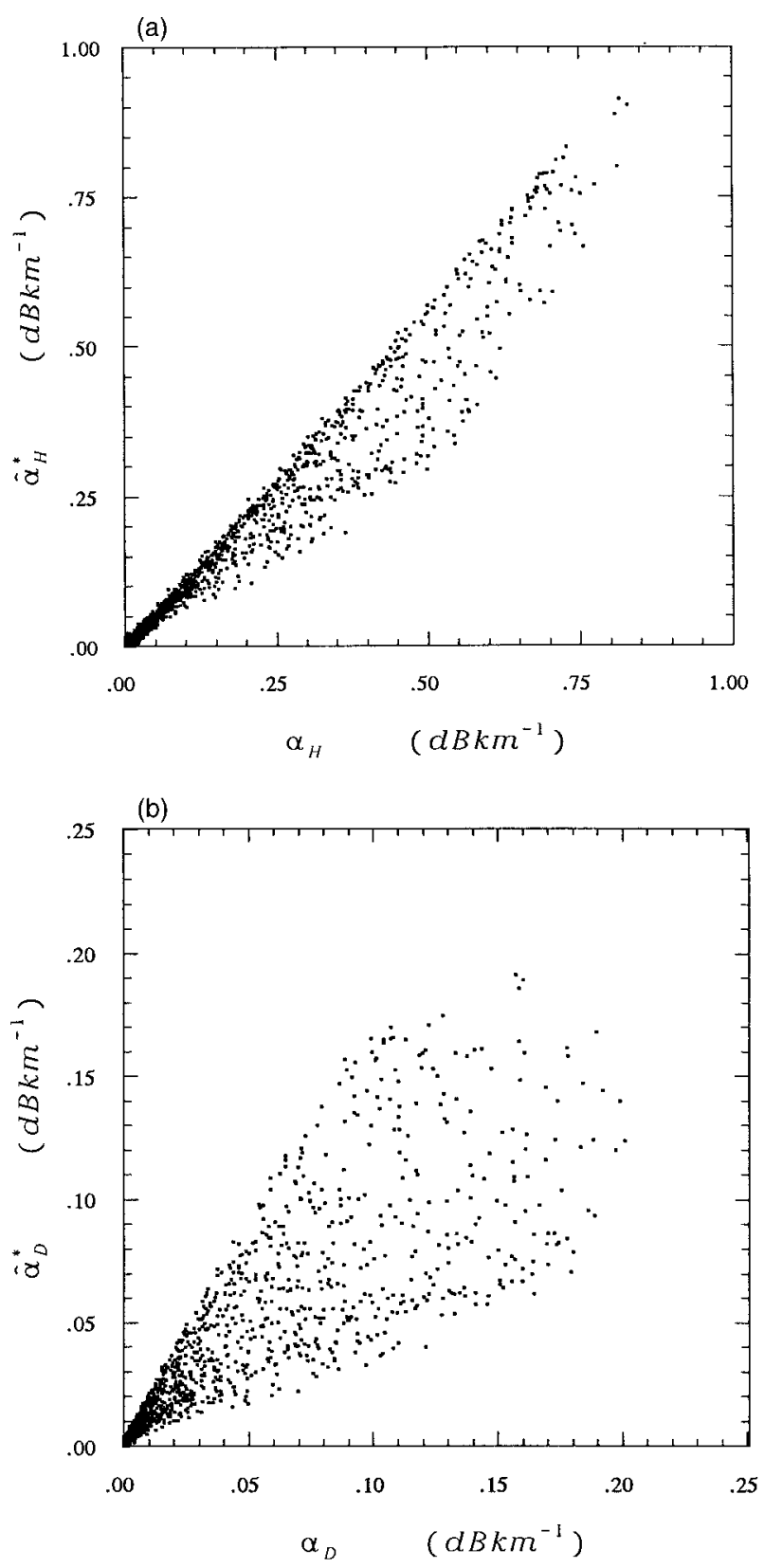

FIG. 3. (a) Scatterplot of the estimate of specific attenuation $\left(\hat{\alpha}_{H}^{*}\right)$ using $K_{\mathrm{DP}}$ as a function of specific attenuation $\left(\hat{\alpha}_{H}\right)$ for different RSDs. (b) Scatterplot of the estimate of specific differential attenuation $\left(\hat{\alpha}_{D}^{*}\right)$ using $K_{\mathrm{DP}}$ as a function of specific differential attenuation $\left(\alpha_{D}\right)$ for different RSDs. 
TABLE 5. Fractional standard error in the estimation of the different algorithms due to the parameterization (first column) and including measurement errors averaged over a $12-\mathrm{km}$ path (second column).

\begin{tabular}{ccc}
\hline \hline Algorithm & FSE $(\%)$ & FSE $(\%)$ \\
\hline$\hat{\alpha}_{H}$ & 11.9 & 10.9 \\
$\hat{\alpha}_{D}$ & 24.1 & 19.1 \\
$\hat{\alpha}_{Z}$ & 38.4 & 34.3 \\
$\hat{\alpha}_{H}^{*}$ & 27.5 & 26.9 \\
$\hat{\alpha}_{D}^{*}$ & 77.8 & 65.2 \\
\hline
\end{tabular}

shift $\left(\Phi_{\mathrm{DP}}\right)$, attenuation $\left(A_{H}\right)$, and differential attenuation $\left(A_{D}\right)$ can be expressed as

$$
\begin{gathered}
\Phi_{\mathrm{DP}}=2 \int_{0}^{R_{c}} K_{\mathrm{DP}}(r) d r, \\
A_{H}=2 \int_{0}^{R_{c}} \alpha_{H}(r) d r,
\end{gathered}
$$

and

$$
A_{D}=2 \int_{0}^{R_{c}} \alpha_{D}(r) d r,
$$

where $R_{c}$ is the range to the observation cell. Since $\alpha_{H}$ and $\alpha_{D}$ are nearly related to $K_{\mathrm{DP}}$ in rainfall, we can estimate $A_{H}$ and $A_{D}$ directly from $\Phi_{\mathrm{DP}}$ measurements, which can be used to correct attenuation and differential attenuation. In the following section we study the error structure of the above-mentioned attenuation correction procedures.

\section{Error structure of attenuation correction procedures}

There are potentially two sources of errors that can affect these correction algorithms, namely, (a) random measurement fluctuations and (b) error in the absolute gain of the radar system (which results as a bias in the estimate of $Z_{H}$ ). The effect of random measurement fluctuations is analyzed in this paper using radar signal simulations. Algorithms (7), (9), and (12) as well as (8) and (13) can be used to estimate $\alpha_{H}$ and $\alpha_{D}$, respectively. Table 5 summarizes the accuracies in the estimation of specific and differential attenuation using the various techniques discussed in this paper. The accuracy is described by the fractional standard error (FSE), which is defined as the standard error normalized with respect to the mean. The first column in Table 5 shows the accuracy in the algorithms due to the parameterization process. We can see in Table 5 that $\hat{\alpha}_{H}, \hat{\alpha}_{H}^{*}$, and $\hat{\alpha}_{Z}$ can estimate $\alpha_{H}$ to an accuracy of $11.9 \%, 27.5 \%$, and $38.4 \%$, respectively. The above error estimates were obtained by averaging over many different RSDs (Ulbrich 1983). Similarly $\hat{\alpha}_{D}$ and $\hat{\alpha}_{D}^{*}$ can estimate $\alpha_{D}$ to an accuracy of $24.1 \%$ and $77.8 \%$, respectively. We need to note here that the above accuracies are reported in the absence of measurement error. However, measurement errors play a significant role in the error structure of all algorithms. We have used radar system simulations (Chandrasekar et al. 1986) to study the effect of measurement errors on the attenuation correction algorithms. The principal assumptions in our simulation are as follows: Gaussian Doppler spectrum with spectrum width of $2 \mathrm{~m} \mathrm{~s}^{-1}$, pulse repetition time of $1 \mathrm{~ms}$, sample pairs number of 64 , wavelength of $5.5 \mathrm{~cm}$, and cross correlation between the horizontal and vertical polarized return signals $\rho_{H, V}$ of 0.99 . We note here that $Z_{H}$ and $Z_{\mathrm{DP}}$ are point measurements (measured at each range gate), whereas $K_{\mathrm{DP}}$ is estimated as the slope of the $\Phi_{\mathrm{DP}}$ range profile. For estimating $K_{\mathrm{DP}}$ we have assumed a uniform path. We have considered two pathlengths, namely, 6 and $12 \mathrm{~km}$, corresponding to 20 and 40 gates with a gate spacing of $300 \mathrm{~m}$. The second column in Table 5 shows the error in the algorithms in the presence of measurement errors after averaging over a $12-\mathrm{km}$ path. We can see that $\hat{\alpha}_{H}$ and $\hat{\alpha}_{D}$ can estimate $\alpha_{H}$ and $\alpha_{D}$ to an accuracy of $11 \%$ and $19 \%$, respectively. Similarly, $\hat{\alpha}_{H}^{*}$ and $\hat{\alpha}_{D}^{*}$ with $K_{\mathrm{DP}}$ estimated averaging over a $12-\mathrm{km}$ path can estimate $\alpha_{H}$ and $\alpha_{D}$ to an accuracy of $27 \%$ and $65 \%$, respectively. In addition, $\hat{\alpha}_{Z}$ can estimate $\alpha_{H}$ to an accuracy of $35 \%$. Since a $12-\mathrm{km}$ path may be considered too long for uniform precipitation approximation, we have repeated the computations for a $6-\mathrm{km}$ path and the results were similar. Note here that even though we have used 6- and $12-\mathrm{km}$ paths to estimate an average specific attenuation for analysis purpose, it does not mean that the attenuation correction is affected. The cumulative attenuation due to the rain cell along a path is represented by an average value over the path. This is done strictly to reduce statistical errors in comparisons, and the attenuation correction procedure can be applied to any pathlength. Table 5 provides an idea about the average accuracy of the attenuation correction procedure. However, the accuracy also changes with the value of specific attenuation. Figure 4 shows the FSE in the estimates $\hat{\alpha}_{H}, \hat{\alpha}_{H}^{*}$, and $\hat{\alpha}_{Z}$ as a function of $\alpha_{H}$. The FSEs are obtained for a $12-\mathrm{km}$ path, including the effect of measurement errors. We can see in Fig. 4 that the specific attenuation can be estimated to an accuracy between $5 \%$ and $25 \%$ when $\alpha_{H}>0.2 \mathrm{~dB} \mathrm{~km}^{-1}$. Among the algorithms to estimate specific attenuation, $\hat{\alpha}_{H}$ has the lowest error. However, the estimate $\hat{\alpha}_{H}^{*}$ has an important advantage that it is not at all affected by errors in radar calibration. Large absolute calibration errors can affect the $\hat{\alpha}_{H}$ estimates significantly, as discussed by Aydin et al. (1989). Therefore, it is important to ensure that the radar is well calibrated before applying reflectivitybased attenuation correction algorithms. Another point to note in Fig. 4 is that, when specific attenuation is negligible $\left(<0.05 \mathrm{~dB} \mathrm{~km}^{-1}\right.$, light rain), the accuracy of $\hat{\alpha}_{Z}$ and $\hat{\alpha}_{H}$ estimates is comparable. Figure 5 shows the FSE of $\hat{\alpha}_{D}$ and $\hat{\alpha}_{D}^{*}$ as a function of $\alpha_{D}$, respectively. The result of Fig. 5 was obtained assuming a pathlength of $12 \mathrm{~km}$, including the effect due tob measurement errors. For correcting differential attenuation we can see that 


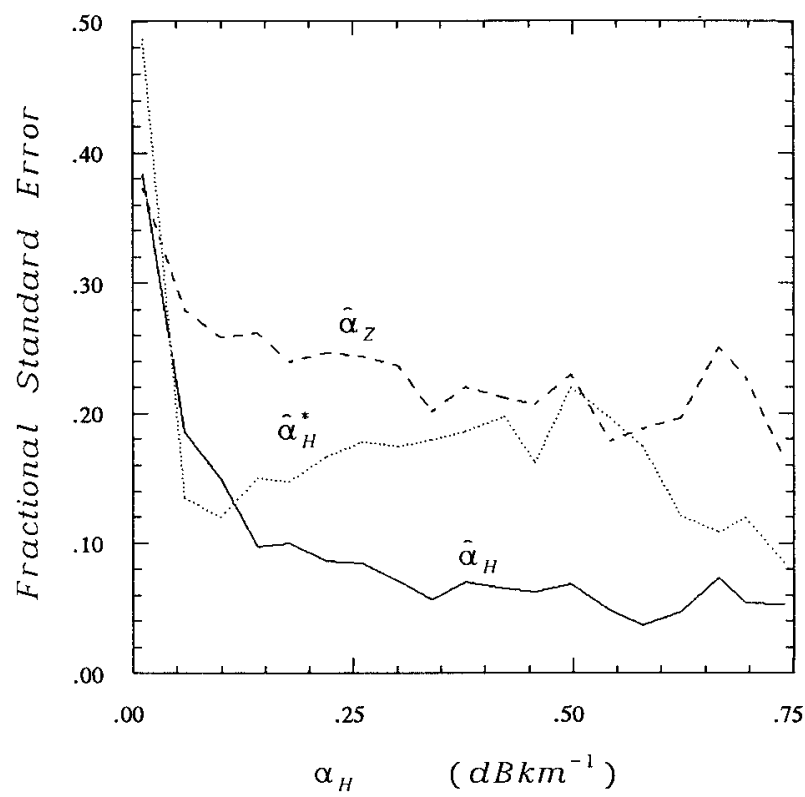

FIG. 4. Fractional standard error of $\hat{\alpha}_{H}, \hat{\alpha}_{H}^{*}$, and $\hat{\alpha}_{z}$ given as a function of specific attenuation $\alpha_{H}$. The estimates were obtained over a $12-\mathrm{km}$ path.

$\hat{\alpha}_{D}$ is significantly more accurate than $\hat{\alpha}_{D}^{*}$. It can be seen in Fig. 5 that $\alpha_{D}$ can be estimated to an accuracy of $10 \%-15 \%$ using algorithm $\hat{\alpha}_{D}$ and $40 \%$ using algorithm $\hat{\alpha}_{D}^{*}$ when $\alpha_{D}>0.05 \mathrm{~dB} \mathrm{~km}^{-1}$. Once again we need to note that $\hat{\alpha}_{D}^{*}$ is immune to absolute calibration errors.

\section{Radar data description}

The radar data used in this paper were collected by the polarimetric C-band Doppler radar POLDIRAD operated by DLR. Details on POLDIRAD can be found in Schroth et al. (1988). The data were collected as part of the collaborative program between IFA of CNR of Italy and the DLR Institute of Atmospheric Physics of Germany. The dataset analyzed here was collected on 29 August 1995 during the passage of a cold front from the north, providing an excellent opportunity to observe the storm over an extended period of time. This cold front stalled over the Alps, leading to long-lasting precipitation on the northern Alpine foreland. The radar data were collected in a plan position indicator (PPI) mode, and the multiparameter radar variables $Z_{H}, Z_{\mathrm{DR}}$, and $\Phi_{\mathrm{DP}}$ were measured by averaging 64 sample pairs at a pulse repetition frequency of 1200 . The data used in this paper were collected at $1^{\circ}$ elevation, and the data analyzed were confined to ranges less than $50 \mathrm{~km}$. This ensured that there was no contamination by the melting layer. The attenuation correction procedures described in section 2 were applied to the radar data and $\hat{\alpha}_{H}$, $\hat{\alpha}_{H}^{*}, \hat{\alpha}_{Z}, \hat{\alpha}_{D}$, and $\hat{\alpha}_{D}^{*}$ were estimated.

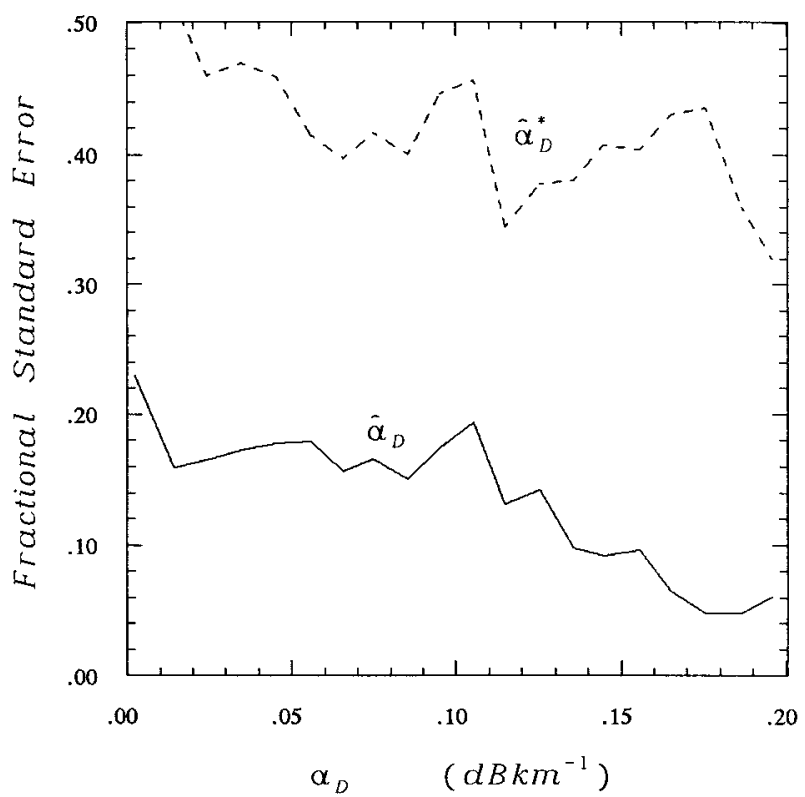

FIG. 5. Fractional standard error of $\hat{\alpha}_{D}$ and $\hat{\alpha}_{D}^{*}$ given as a function of specific diffberential attenuation $\alpha_{D}$. The estimates were obtained over a $12-\mathrm{km}$ path.

\section{Experimental results}

For comparative analysis the radar data of each measured ray were divided into intervals of $12-\mathrm{km}$ pathlength. In each 12-km segment $K_{\mathrm{DP}}$ was estimated using a straight line fit to the $\Phi_{\mathrm{DP}}$ profile. Subsequently, average values of $\hat{\alpha}_{H}, \hat{\alpha}_{H}^{*}$, and $\hat{\alpha}_{Z}$ as well as $\hat{\alpha}_{D}$ and $\hat{\alpha}_{D}^{*}$ were computed. Figure 6a shows a comparison of $\hat{\alpha}_{H}$ versus $\hat{\alpha}_{H}^{*}$. The vertical bars indicate the standard deviation about the mean value. We can see in Fig. 6a that $\hat{\alpha}_{H}$ and $\hat{\alpha}_{H}^{*}$ agree fairly well with a slope of 0.95 . The bias for the comparison between $\hat{\alpha}_{H}$ and $\hat{\alpha}_{H}^{*}$ was found to be $4 \%$ (very low). The results of Fig. 6a show that the two estimates agree very well. The maximum specific attenuation encountered on a $12-\mathrm{km}$ path was 0.22 $\mathrm{dB} \mathrm{km} \mathrm{km}^{-1}$, which corresponds to a two-way cumulative attenuation of $5.3 \mathrm{~dB}$, thereby indicating the importance of the attenuation correction. Figure $6 \mathrm{~b}$ shows a comparison of $\hat{\alpha}_{Z}$ and $\hat{\alpha}_{H}^{*}$. The vertical bars again indicate standard deviation. We can see in Fig. $6 \mathrm{~b}$ that there is a bias in $\hat{\alpha}_{z}$. This is because the arbitrary $\alpha_{Z}-Z$ relation used in this paper is not the best $\alpha_{Z}-Z$ relation corresponding to the data. The $\alpha_{Z}-Z$ relation exhibits a wide variability very similar to $Z-R$ relations. Figure 7 shows the FSE in the comparison of $\hat{\alpha}_{H}$ and $\hat{\alpha}_{Z}$ as a function of $\hat{\alpha}_{H}^{*}$. Also shown in Fig. 7 is the FSE between $\hat{\alpha}_{H}$ and $\hat{\alpha}_{H}^{*}$ that was computed based on simulation including the effect of measurement errors. We need to note here that the FSE of comparison between $\hat{\alpha}_{H}$ and $\hat{\alpha}_{H}^{*}$ includes error in the estimation of $\alpha_{H}$ and $\alpha_{H}^{*}$. Similarly, the FSE of comparison between $\hat{\alpha}_{Z}$ and $\hat{\alpha}_{H}^{*}$ includes errors in the estimation of $\alpha_{Z}$ and $\alpha_{H}^{*}$. In general a fairly good agreement exists between experimentally observed FSE be- 

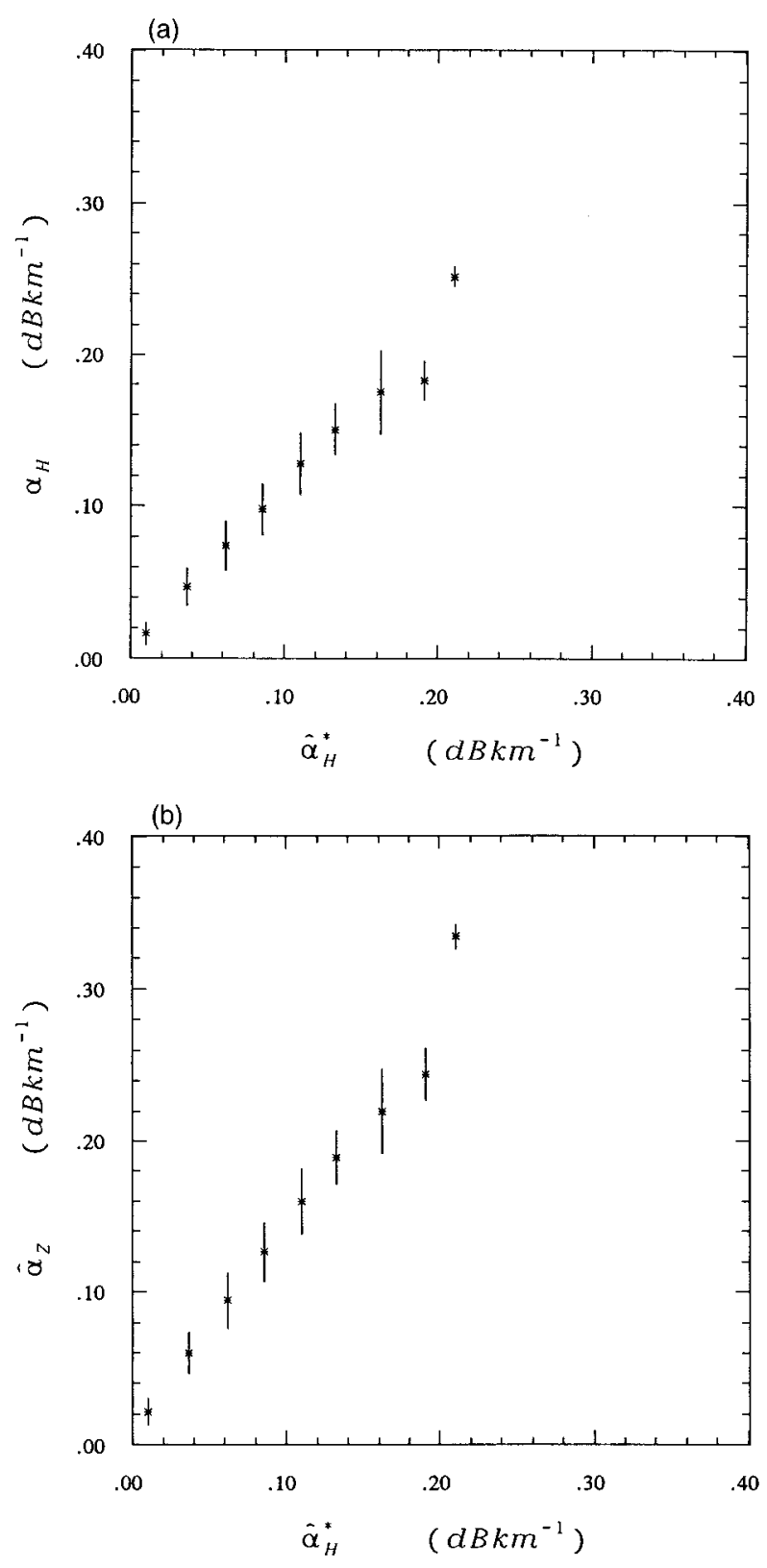

FIG. 6. (a) Comparison of the estimates $\hat{\alpha}_{H}$ and $\hat{\alpha}_{H}^{*}$ from the radar data collected by DLR radar. The dots indicate the mean and the vertical bars denote standard deviation. (b) Comparison of the estimates $\hat{\alpha}_{Z}$ and $\hat{\alpha}_{H}^{*}$ from the radar data collected by DLR radar. The dots indicate the mean and the vertical bars denote standard deviation.

tween $\hat{\alpha}_{H}$ and $\hat{\alpha}_{H}^{*}$ with simulation results. The agreement is not perfect, which can be due to many reasons-one of them can be the difference in the ambient temperature of the environment where data was obtained and the parameterization used. In addition it can be seen in Fig. 7 that the FSE of comparison between $\hat{\alpha}_{Z}$ and $\hat{\alpha}_{H}^{*}$ is higher than the FSE of comparison between $\hat{\alpha}_{H}$ and $\hat{\alpha}_{H}^{*}$. This is due to the biased nature of $\hat{\alpha}_{Z}$, which can be seen in Fig. 6b. Similarly, Fig. 8 shows the com-

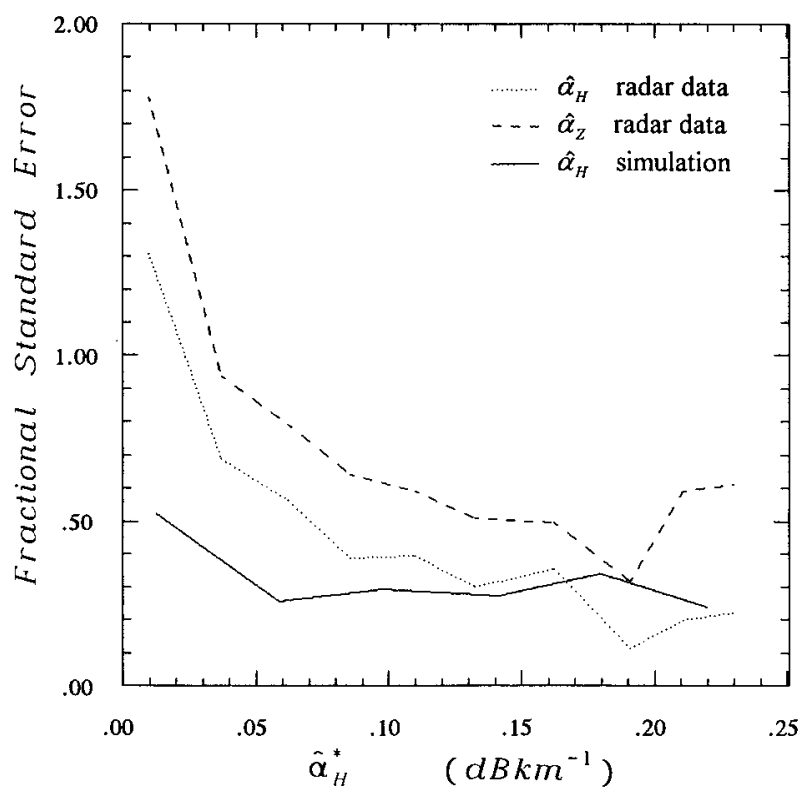

FIG. 7. Fractional standard error of the comparison between $\hat{\alpha}_{H}$ and $\hat{\alpha}_{H}^{*}$ (dotted line) as well as $\hat{\alpha}_{Z}$ and $\hat{\alpha}_{H}^{*}$ (dashed line) shown as a function of $\hat{\alpha}_{H}^{*}$ for the data collected by DLR radar. The solid line shows the FSE of the comparison between $\hat{\alpha}_{H}$ and $\hat{\alpha}_{H}^{*}$ as a function of $\hat{\alpha}_{H}^{*}$ obtained from simulation.

parison of $\hat{\alpha}_{D}$ versus $\hat{\alpha}_{D}^{*}$. It can be seen in Fig. 8 that the two estimates are of the same order and agree reasonably well. The vertical bars indicate standard deviation in the comparison. Figure 9 shows the FSE in the comparison of $\hat{\alpha}_{D}$ and $\hat{\alpha}_{D}^{*}$. Also shown in Fig. 9 is the FSE between $\hat{\alpha}_{D}$ and $\hat{\alpha}_{D}^{*}$ that was computed based on simulation including the effect of measurement error.

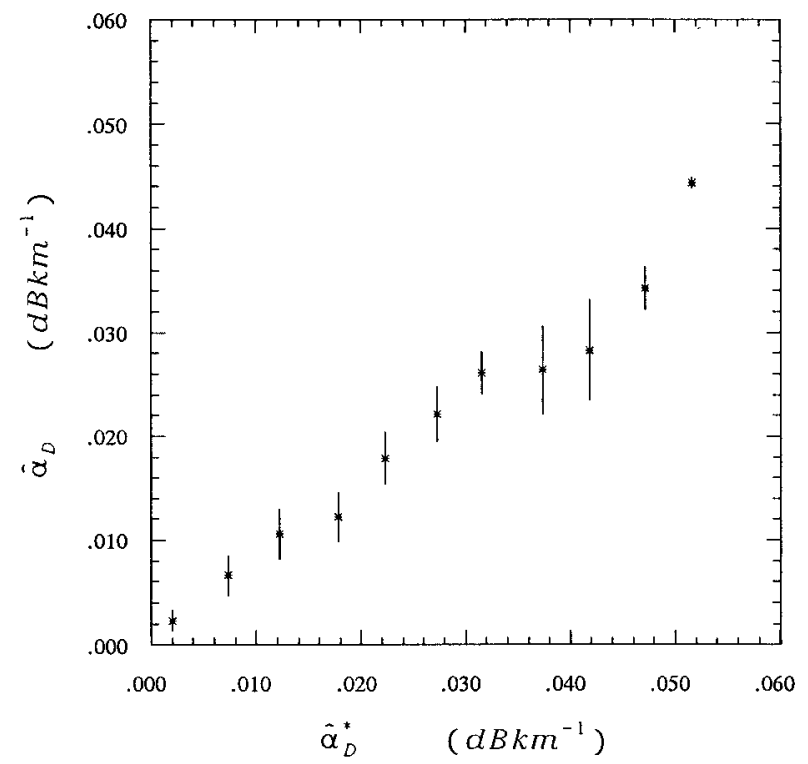

FIG. 8. Comparison of the estimates $\hat{\alpha}_{D}$ and $\hat{\alpha}_{D}^{*}$ from radar data collected by DLR radar. The dots indicate the mean and the vertical bars indicate the standard deviation. 


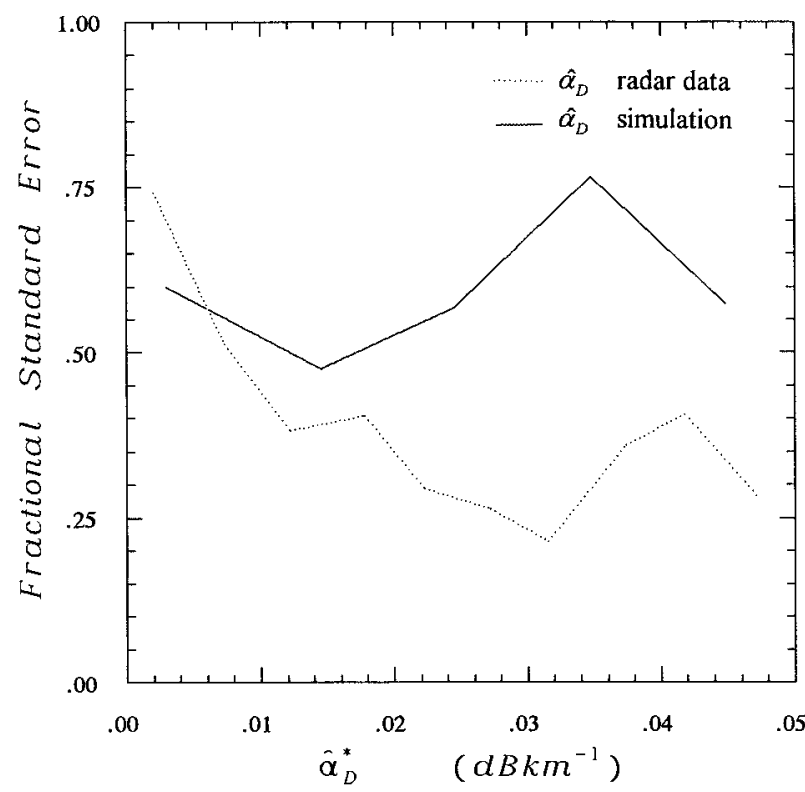

FIG. 9. Fractional standard error of the comparison between $\hat{\alpha}_{D}$ and $\hat{\alpha}_{D}^{*}$ (dotted line) shown as a function of $\hat{\alpha}_{D}^{*}$ for the data collected by DLR radar. The solid line shows the FSE of the comparison between $\hat{\alpha}_{D}$ and $\hat{\alpha}_{D}^{*}$ as a function of $\hat{\alpha}_{D}^{*}$ obtained from simulation.

Note here that the FSE of comparison between $\hat{\alpha}_{D}$ and $\hat{\alpha}_{D}^{*}$ includes errors in the estimation of $\alpha_{D}$ and $\alpha_{D}^{*}$. The experimentally observed FSE between $\hat{\alpha}_{D}$ and $\hat{\alpha}_{D}^{*}$ is of the same order as that from simulation. The agreement is not as good as that between $\hat{\alpha}_{H}$ and $\hat{\alpha}_{H}^{*}$, primarily because $\hat{\alpha}_{D}$ and $\hat{\alpha}_{D}^{*}$ have more scatter in the parameterization process compared to estimates of $\alpha_{H}$.

\section{Summary and conclusions}

Algorithms to correct for attenuation at C-band frequencies are analyzed. C-band radar measurements of reflectivity are affected by absolute attenuation and the measurements of differential reflectivity are affected by differential attenuation. Quantitative application of echo power requires correction for attenuation and differential attenuation. Two attenuation correction algorithms, namely, one using $Z_{H}$ and $Z_{\mathrm{DR}}$ and the other using $K_{\mathrm{DP}}$, are studied based on theoretical analysis as well as data collected by a C-band dual-polarized radar. Theoretical analysis shows that the algorithm to correct for attenuation based on $Z_{H}$ and $Z_{\mathrm{DR}}$ can correct attenuation to an accuracy of $10 \%$ when the specific attenuation is more than $0.2 \mathrm{~dB} \mathrm{~km}^{-1}$. Similarly, the algorithm to correct attenuation based on $K_{\mathrm{DP}}$ can correct attenuation to an accuracy of $20 \%$. Though the algorithm $\hat{\alpha}_{H}^{*}$ has only half the accuracy of $\hat{\alpha}_{H}$, it has the advantage that it is immune to radar calibration errors. A large error in radar calibration can significantly deteriorate the performance of $\hat{\alpha}_{H}$. The algorithm based on $Z_{H}$ (arbitrarily chosen) corrected attenuation to an accuracy of $30 \%$. This accuracy was achieved after ensuring that there was no bias in the reflectivity-based algorithms. However, this is not possible in practical application. The algorithm to correct differential attenuation based on $Z_{H}$ and $Z_{\mathrm{DR}}\left(\hat{\alpha}_{D}\right)$ can correct to an accuracy of $10 \%$ on the average. Similarly, the algorithm to correct differential attenuation based on $K_{\mathrm{DP}}\left(\hat{\alpha}_{D}^{*}\right)$ can correct to an accuracy of $40 \%$ on the average. Once again, in spite of the reduced accuracy of $\hat{\alpha}_{D}^{*}$, the algorithm has the advantage of being immune to absolute calibration errors. The above-mentioned accuracies were obtained theoretically, including the effects of measurement errors. Direct verification of the above results is nearly impossible and indirect procedures are used to verify the accuracy in the attenuation correction procedure.

Data collected by the C-band dual-polarized radar operated by DLR were utilized to verify the attenuation correction procedures. Theoretical evaluation indicated that the two attenuation correction procedures, namely, $\hat{\alpha}_{H}$ and $\hat{\alpha}_{H}^{*}$, should agree with each other in the mean. In addition the error structure of the intercomparison is a combination of the errors in $\hat{\alpha}_{H}$ and $\hat{\alpha}_{H}^{*}$. Theoretical analysis comparing $\hat{\alpha}_{H}$ versus $\hat{\alpha}_{H}^{*}$ shows that the accuracy of the intercomparison is approximately 20\%-30\%. Similarly theoretical analysis yielded that algorithms to correct for differential attenuation, namely, $\hat{\alpha}_{D}$ and $\hat{\alpha}_{D}^{*}$, can be compared to an accuracy of 30\%-40\%. Data from C-band dual-polarized radar were analyzed to intercompare the two attenuation correction algorithms $\hat{\alpha}_{H}$ and $\hat{\alpha}_{H}^{*}$. The two attenuation correction algorithms $\hat{\alpha}_{H}$ and $\hat{\alpha}_{H}^{*}$ agreed with each other fairly well and the FSE in the intercomparison was between $20 \%$ and $40 \%$ for specific attenuation higher than $0.1 \mathrm{~dB} \mathrm{~km}^{-1}$, which is in excellent agreement with theoretical results presented in this paper. Similarly, the two algorithms to correct the differential attenuation, namely, $\hat{\alpha}_{D}$ and $\hat{\alpha}_{D}^{*}$, compared fairly well with each other. Thus the intercomparison study of the attenuation correction algorithms based on radar data provides an indirect verification of the theoretical results on the accuracies of the algorithms.

Acknowledgments. This research was supported partially by the Nationalb Group for Defense from Hydrological Hazard (CNR, Italy), by Progetto Strategico Mesoscale Alpine Programme (CNR, Italy), COST-75 (STSM), and by NASA (TRMM). The authors are grateful to P. Iacovelli for assistance rendered during the preparation of the manuscript.

\section{REFERENCES}

Aydin, K., Y. Zhao, and T. A. Seliga, 1989: Rain-induced attenuation effects on C-band dual-polarization meteorological radars. IEEE Trans. Geosci. Remote Sens., 27, 57-66.

Beard, K. V., and C. Chuang, 1987: A new model for the equilibrium shape of raindrops. J. Atmos. Sci., 44, 1509-1524.

Bringi, V. N., and A. Hendry, 1990: Technology of polarization diversity radars for meteorology. Radar in Meteorology, D. Atlas, Ed., Amer. Meteor. Soc., 153-190. 
V. Chandrasekar, N. Balakrishnan, and D. S. Zrnić, 1990: An examination of propagation effects in rainfall on radar measurements at microwave frequencies. J. Atmos. Oceanic Technol., 7, 829-840.

Chandrasekar, V., V. N. Bringi, and P. J. Brockwell, 1986: Statistical properties of dual-polarized radar signals. Preprints, 23d Conf. on Radar Meteorology, Snowmass, CO, Amer. Meteor. Soc., 193-196.

, W. A. Cooper, and V. N. Bringi, 1988: Axis ratios and oscillations of raindrops. J. Atmos. Sci., 45, 1323-1333.

Gorgucci, E., G. Scarchilli, and V. Chandrasekar, 1995: Radar and raingage measurements of rainfall over the Arno basin. Preprints, Conf. on Hydrology, Dallas, TX, Amer. Meteor. Soc., 68-73.
Hildebrand, P. H., 1978: Iterative correction for attenuation of $5 \mathrm{~cm}$ radar in rain. J. Appl. Meteor., 17, 508-514.

Scarchilli, G., E. Gorgucci, V. Chandrasekar, and T. A. Seliga, 1993: Rainfall estimation using polarimetric techniques at C-band frequencies. J. Appl. Meteor., 32, 1150-1159.

Schroth, A., M. Chandra, and P. F. Meischner, 1988: A C-band coherent polarimetric radar for propagation and cloud physics research. J. Atmos. Oceanic Technol., 5, 803-822.

Seliga, T. A., and V. N. Bringi, 1976: Potential use of the radar reflectivity at orthogonal polarizations for measuring precipitation. J. Appl. Meteor., 15, 69-76.

Ulbrich, C. W., 1983: Natural variations in the analytical form of raindrop size distributions. J. Climate Appl. Meteor., 22, 17641775. 